\title{
Isolation and Characterization of Bacteria of Mangrove Rhizosphere in the Mekong Delta, Vietnam
}

\author{
Ho Thanh Tam \\ Can Tho College, Can Tho City, Vietnam \\ Cao Ngoc Diep \\ Dept. Microbiology Biotechnology, Biotechnology \\ $R \& D$ Institute, Can Tho University, Can Tho City, Vietnam
}

\begin{abstract}
Total of 125 rhizospheric bacterial isolates were isolated from 18 rhizospheric soil samples of mangrove at Ca Mau Peninsula (Ca Mau province, Mekong Delta of Vietnam). Although all of them had the abilities of ammonium synthesis and phosphate solubilization, the isolated strains had excellent phosphate solubilizing potential. The sequences from selected nitrogen-fixing and phosphate-solubilizing bacteria ( 22 isolates) showed high degrees of similarity to those of the GenBank reference strains (between $97 \%$ and $99 \%$ ). From 22 isolates, 16 strains belonged to Bacilli, and 6 strains were Gamma-Proteobacteria. Whereas our results showed that there were some good strains for nitrogen fixation as Bacillus subtilis DLB4b, Bacillus sp. VAB2b, Enterobacter sp. MAB1b and Bacillus sp. MDB1c and strains for phosphate solubilation as Bacillus subtilis MLN1b, Bacillus sp. MLN1c, Bacillus subtilis VDN1f and Bacillus subtilis MDN1c, all of them tolerated at a concentration of $4 \%$ NaCl. However, the strain Bacillus subtilis MAB2b revealed as a promising candidate with multiple beneficial characteristics (both good nitrogen fixation and phosphate solubilization). Besides, the isolated bacterial strain has the potential for application as inoculants adapted to poor, soil salinity as well many kinds of crop because it is not only famous strain but also safe strain for sustainable agriculture in "sea level rise" condition.
\end{abstract}

Keywords: 16S rRNA Gene Sequence, Mangrove Rhizosphere Bacteria, Nitrogen Fixation, Phosphate Solubilization, soil salinity

\section{INTRODUCTION}

Mangroves are unique coastal plants which have originated due to the tectonic land shifts because of which terrestrial plants got bared to the open sea with ecological and economic importance. They not only provide socio-economic benefits to local tribes, but also provide protection to coastal areas against natural disasters and facilitate the formation of land by trapping sediments [1][2]. Around 34 major and 20 minor mangrove species belonging to about 20 genera in over 11 families have been recorded globally [3]. Mangroves constitute a significant part of tropical coastal biodiversity which occupy less than $1 \%$ of the world's surface [4] and are mainly found between the Tropic of Cancer and the Tropic of Capricorn on all continents covering an estimated $75 \%$ of the tropical coastline worldwide. Mangroves of South and Southeast Asia form the most extensive and diverse mangrove system comprising $41.4 \%$ of global mangroves in the world [5].

Bacterial diversity from these ecosystems has been studied worldwide for their unique biochemical processes. The present study includes isolation, morphological characterization and identification of rhizospheric bacteria using biochemical and molecular biology techniques [6] [7]. Molecular biology techniques like 16S rRNA techniques are an important tool in final identification of bacteria sequencing this gene, and provide genus and species identification for isolates that do not fit any recognized biochemical profiles. It gives acceptable identification which otherwise according to conventional system of taxonomy is not possible [8].

Some studies are available for the beneficial bacteria associated with the natural mangrove habitats [9] [10] [11] [12] [13]. However, no such studies are available for artificially developed mangrove habitats. In mangrove ecosystems, high rates of nitrogen fixation have been associated with dead and decomposing leaves [14] , pneumatophores [15] [16] and the rhizosphere soil [10]. $\mathrm{N}_{2}$ fixation in mangrove sediments is likely to be limited by insufficient energy sources. The low rates of $\mathrm{N}_{2}$ fixation by heterotrophic bacteria detected in marine water are probably due to lack of energy sources. Phosphorous is one of the major plant nutrients, second only to nitrogen [17], so phosphate-solubilizing microorganisms (PSMs) play an important role in supplementing phosphorus to plants and allowing the sustainable use of phosphate fertilizers [18]. Fungi and inorganic phosphate-solubilizing bacteria present in the mangrove rhizosphere participate in releasing soluble phosphate into pore water [12]. Certain bacteria exhibit high phosphatase activity, capable of solubilizing phosphate [19]. However, very little information is available about beneficial bacterial diversity [9] and their activity in mangrove soil of Vietnam. Therefore, the aims of this study were (i) to isolate nitrogen-fixing bacteria and phosphate-solubilizing bacteria, (ii) to obtain their characterization as salt-tolerance, colonies....and (iii) to identify by $16 \mathrm{~S}$ rDNA techniques. 


\section{A. Collect of samples}

\section{MATERIALS AND METHODS}

Soil samples adhering to the root system were collected carefully from four species of mangroves viz. Rhizophora mucronata, Bruguiera cylindrica, and Avicennia marina from a 5 year old plantation site, raised along the CaMau Peninsula (Lat. 09 $05^{\prime} 10^{\prime}$ N; Long. $105^{\circ} 15^{\prime} 00^{\prime}$ E), located at the end of the Mekong Delta (Vietnam) (Figure 1).
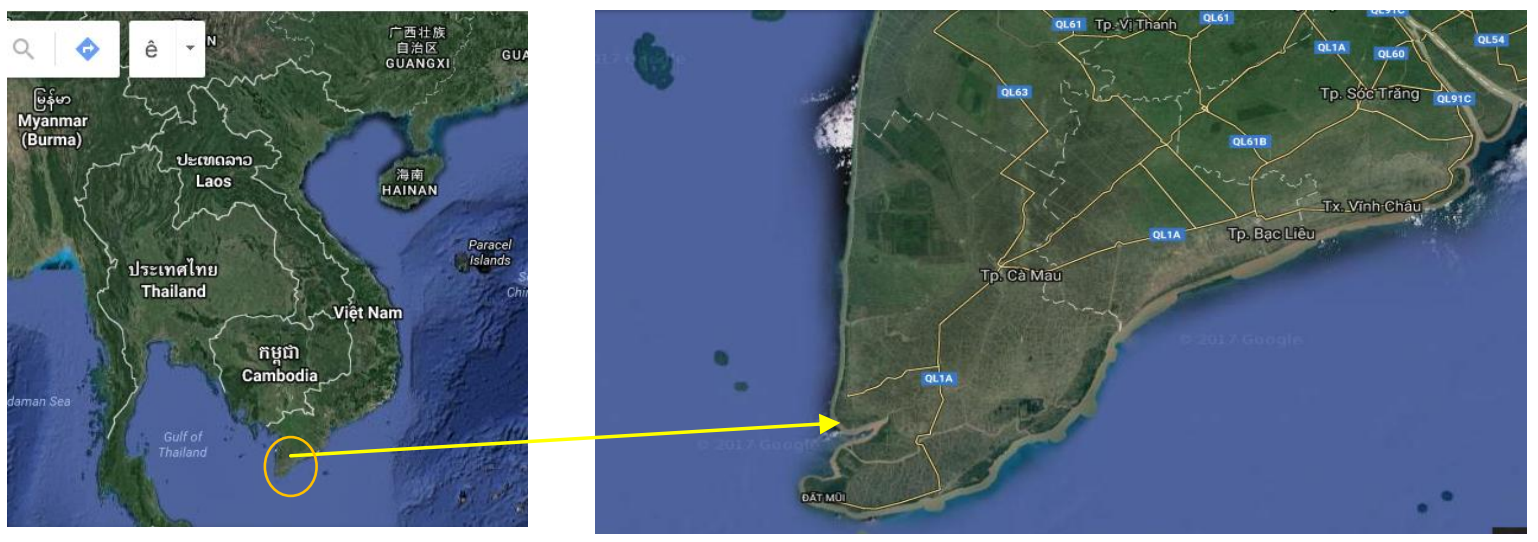

Figure 1. Sample collection sites in Ca mau Peninsula, Ca Mau province, Vietnam (the Mekong Delta, Vietnam)

The samples were collected in December, 2015. For isolation of bacterial rhizosphere samples were collected during the low tide and brought to the laboratory immediately for analyses within $3 \mathrm{~h}$. Soil samples were collected by using a sterile spatula and stored in sterile polythene bags.

A known weight of soil (1 g) was aseptically weighed and transferred to a stoppered $(150 \mathrm{~mL})$ sterile conical flask containing $99 \mathrm{~mL}$ of sterile diluent. The sediment-diluent mixture was agitated by means of mechanical shaking for about $10 \mathrm{~min}$. and later subjected to bacteriological examination.

One hundred microliters from appropriate dilutions were plated on two different media, viz Burk's N free agar plus $2 \% \mathrm{NaCl}$ [20] and NBRIP agar plus $2 \% \mathrm{NaCl}$ [21] and they were kept to refrigerator for counting by viable drop plate count [22] (Hoben and Somasegaran, 1982) and isolation of nitrogen-fixing bacteria in Burk'N free media plus $2 \% \mathrm{NaCl}$ [20] and phosphate-solubilizing bacteria in NBRIP media plus $2 \% \mathrm{NaCl}$ [21]; Cultures were streaked on media to obtain single colonies. To check for phosphate solubilization ability or nitrogen fixation ability, colonies from Burk'N free media were streaked to NBRIP media and colonies from NBRIP media were also cultivated to Burk's $\mathrm{N}$ free media in order to select the colonies which developed on two media (or microbes having $\mathrm{N}_{2}$-fixing and phosphate-solubilizing ability).

\section{B. Morphological Characterization}

The morphological characterization of the bacterial colonies were carried out according to on the basis of their shape, size, colour, margin, elevation on the media and Gram staining were performed to decide the further determinative protocol. All isolates were tested on media (Burk's or NBRIP) with higher NaCl concentration $(2.5$ to $4.0 \% \mathrm{NaCl})$.

\section{Screening for Biofertilizer Activities}

The ability to fix $\mathrm{N}_{2}$ was tested on Burk' $\mathrm{N}$-free liquid medium incubating at $30^{\circ} \mathrm{C}$ and the ammonium concentration in medium was measured by Phenol Nitroprusside method after 2,4,6 and 8 days inoculated (DAI). Besides, inorganic phosphate solubilizing ability was tested on NBRIP liquid medium, incubated at $30^{\circ} \mathrm{C}$ and the $\mathrm{P}_{2} \mathrm{O}_{5}$ concentration was measured by ammonium molypdate method.

\section{Molecular Analysis}

Genomic DNA Isolation

Culture was centrifuged at 10,000 rpm for $5 \mathrm{~min}$. Pellet was collected and resuspended by adding $9 \mathrm{ml}$ of STE buffer $(0.1 \mathrm{mM} \mathrm{NaCl}, 10 \mathrm{mM}$ Tris, $10 \mathrm{mM}$ EDTA) $1 \mathrm{ml}$ of SDS (10\% Stock Solution). The suspension was incubated at $70^{\circ} \mathrm{C}$ for $1 \mathrm{hr}$. and centrifuged at $6000 \mathrm{rpm}$ for $10 \mathrm{~min}$ at room temperature. The supernatant was collected in fresh tube and add equal volume of Phenol:Chloroform:Isoamyl alcohol (PCI mix) (25:24:1) was added and mixed slowly. The suspension was centrifuged at $6000 \mathrm{rpm}$ for $10 \mathrm{~min}$. The aqueous phase in fresh tube.

Equal vol. of Chloroform: Isoamyl alcohol (24:1) and mix slowly and centrifuged at $6000 \mathrm{rpm}$ for $10 \mathrm{~min}$. The aqueous phase was collected and added double the vol. of absolute alcohol was added. The tube was subjected 
to overnight incubation in $-20^{\circ} \mathrm{C}$. The solution was centrifuged at $6000 \mathrm{rpm} 4^{\circ} \mathrm{C}$ for $10 \mathrm{~min}$ and the pellet was resuspended in 1/10th $\mathrm{ml}$ of $3 \mathrm{M}$ sodium acetate and $10 \mathrm{ml}$ of absolute alcohol and centrifuged at $6000 \mathrm{rpm} 4^{\circ} \mathrm{C}$ for $10 \mathrm{~min}$. The supernatant was discarded and the pellet was air dried. The pellet was dissolved in $1 \mathrm{ml}$ sterile TE buffer. The DNA quality was checked using Agarose gel electrophoresis and quantified using Nanodrop.

PCR Amplification and Phylogenetic Analysis

Amplification of $16 \mathrm{~S}$ rDNA by PCR was carried out using the universal primers $8 \mathrm{~F}$ and 1492R [23]. The $50 \mu \mathrm{L}$ reactionmixture consisted of $2.5 \mathrm{U}$ Taq Polymerase (Fermentas), $50 \mu \mathrm{M}$ of each deooxynucleotide triphosphate, $500 \mathrm{nM}$ of each primer (Fermentas) and $20 \mathrm{ng}$ DNA. The thermocycling profide was carried out with an initial denaturation at $95^{\circ} \mathrm{C}(5 \mathrm{~min})$ followed by 30 cycles of denaturation at $95^{\circ} \mathrm{C}(30 \mathrm{~s})$, annealing at $55^{\circ} \mathrm{C}(30 \mathrm{~s})$, extension at $72^{\circ} \mathrm{C}(90 \mathrm{~s})$ and a final extension at $72^{\circ} \mathrm{C}(10 \mathrm{~min})$ in $\mathrm{C} 1000$ Thermal Cycler (Bio-Rad). Aliquots $(10 \mu \mathrm{l})$ of PCR products were electrophoresed and visualized in 1\% agarose gels using standard electrophoresis procedures. Partial 16S rRNA gene of selectived isolates in each group were sequenced by MACROGEN, Republic of Korea (dna.macrogen.com). Finally, 16S rRNA sequence of the isolate was compared with that of other microorganisms by way BLAST (http://www.ncbi.nlm.nih.gov/BLAST/Blast.cgi); In the best isolate(s) (especially high phosphate solubilization ability) were chosen to sequence and the results were compared to sequences of GenBank based on partial 16S rRNA sequences to show relationships between PGPR strains [24] and phylogenetic tree were constructed by the maximum-likelihood method using the MEGA software version 6.05 based on 1000 bootstraps.

Data Analyses

Data from ammonium and orthophosphate concentrations in media were analysed in completely randomized design with three replicates and parameters of pot experiment also was arranged to completely randomized design with seven replications and Duncan test at $\mathrm{P}=0.01$ or $\mathrm{P}=0.05$ were used to differentiate between statistically different means using SPSS version 16.

\section{RESULTS AND DISCUSSION}

\section{A. Soil pH and bacterial population}

Soil pH of mangrove rhizosphere ranged from 5.27 to 6.68 (Table 1) and nitrogen-fixing and phosphatesolubilizing bacterial population in soil were significantly high. Particularly, population of and nitrogen-fixing and phosphate-solubilizing bacteria in rhizosphere soil of Bruguiera cylindrica was the highest, while that of Rhizophora mucronata was the least. A similar trend has been recorded with decomposing leaves of mangroves [25]. The variation recorded presently between Rhizophora and Avicennia species may be attributed to the pattern of root system.

Table 1 - Soil $\mathrm{pH}$ and $\mathrm{N}_{2}$-fixing and phosphate-solubilizing bacterial population in mangrove rhizosphere soil

\begin{tabular}{|c|c|c|c|c|c|c|}
\hline Soil sample site & $\begin{array}{c}\text { Kind of } \\
\text { Plant }\end{array}$ & Soil pH & $\begin{array}{l}\mathrm{N}_{2} \text {-fixing } \\
\text { bacteria } \\
\text { population }\end{array}$ & Average & $\begin{array}{c}\text { P-solubilizing } \\
\text { bacteria } \\
\text { population }\end{array}$ & Average \\
\hline & & & \multicolumn{4}{|c|}{ CFU $\log _{10} / \mathrm{g}$ soil } \\
\hline Phong Dien 1 & \multirow{6}{*}{$\begin{array}{c}\text { Rhizophora } \\
\text { mucronata }\end{array}$} & 6.28 & 6.011 & \multirow{6}{*}{5.356} & 5.637 & \multirow{6}{*}{5.667} \\
\hline Phong Dien 2 & & 6.24 & 5.000 & & 5.452 & \\
\hline Phong Lac 1 & & 5.97 & 5.426 & & 3.921 & \\
\hline Phong Lac 2 & & 6.52 & 5.784 & & 6.125 & \\
\hline Loi An 1 & & 5.81 & 5.720 & & 5.067 & \\
\hline Loi An 2 & & 5.27 & 6.125 & & 5.934 & \\
\hline Phong Dien 3 & \multirow{6}{*}{$\begin{array}{l}\text { Bruguiera } \\
\text { cylindrica }\end{array}$} & 6.31 & 6.861 & \multirow{6}{*}{6.875} & 6.831 & \multirow{6}{*}{6.908} \\
\hline Phong Dien 4 & & 6.11 & 7.151 & & 7.071 & \\
\hline Phong Lac 3 & & 6.32 & 7.102 & & 7.242 & \\
\hline Phong Lac 4 & & 5.91 & 6.671 & & 6.882 & \\
\hline Loi An 3 & & 5.41 & 6.871 & & 6.361 & \\
\hline Loi An 4 & & 5.81 & 6.801 & & 6.764 & \\
\hline Phong Dien 5 & \multirow{6}{*}{$\begin{array}{l}\text { Avicennia } \\
\text { marina }\end{array}$} & 6.55 & 6.284 & \multirow{6}{*}{6.258} & 6.493 & \multirow{6}{*}{5.832} \\
\hline Phong Dien 6 & & 6.55 & 5.962 & & 6.448 & \\
\hline Phong Lac 5 & & 6.68 & 4.875 & & 6.875 & \\
\hline Phong Lac 6 & & 6.60 & 6.088 & & 6.489 & \\
\hline Loi An 5 & & 6.76 & 6.253 & & 6.004 & \\
\hline Loi An 6 & & 6.50 & 5.530 & & 5.243 & \\
\hline
\end{tabular}


Avicennia produces numerous, soft aerial roots (pneumatophores) that may flush the soil with oxygen, providing a congenial environment for aerobic azotobacters [26]. However our result showed that there were the highest population of nitrogen-fixing and phosphate-solubilizing bacteria in rhizosphere soil of Bruguiera cylindrica, this can explain the contribution of this kind of plant as leaves and roots into soils and this increased organic matter and other nutrients into soil so that there was a significant linear relationship between phosphatesolubilizing bacteria population with soil $\mathrm{pH}$ at $\mathrm{P}<0.01$ in rhizosphere soil of Bruguiera cylindrica (Table 2). Soil $\mathrm{pH}$ decreased but phoshate-solubilizing bacterial population increased noticeably $(\mathrm{P}>0.01)$ perhaps these $\mathrm{P}$ solubilizing bacterial strains released organic acids to dissolve non-soluble $\mathrm{P}$ in soils to soluble $\mathrm{P}$. Bacteria solubilise phosphate in areas where the soil is oxygenated (e.g., near the mangrove roots) and may, therefore, serve an important role in P uptake by the plant [27]. It is generally accepted that the mechanism of mineral phosphate solubilization by phosphate solubilising bacteria (PSB) is associated with the release of low molecular weight organic acids [28], which through their hydroxyl and carboxyl group chelate the cations bound to phosphate, their by converting it into soluble form [29].

One hundred and twenty five bacterial isolates, included 37, 45 and 43 isolates from rhizoshere soil of Rhizophora mucronata, Bruguiera cylindrica and Avicennia marina respectively, were isolated from 18 soil samples in two media (Burk'N free and NBRIP medium) (Table 3) and all isolates grew well on both of media (they have nitrogen fixation and phosphate solubilizing abilities) (Figure 2a and Figure 2b).

Table 2 - The relationship between population of $\mathrm{N}_{2}$-fixing and phosphate-solubilizing bacteria with soil $\mathrm{pH}$ in mangrove soil

\begin{tabular}{|l|l|l|}
\hline \multirow{2}{*}{ Soil $\mathrm{pH}$} & \multicolumn{2}{|c|}{ Population (cfu/dry soil gramme) } \\
\cline { 2 - 3 } & \multicolumn{1}{|c|}{$\mathrm{N}_{2}$-fixing bacteria } & \multicolumn{1}{c|}{ Phosphate-solubilizing bacteria } \\
\hline \multirow{3}{*}{ Rhizophora mucronata } & $\mathrm{r}=0.375(\mathrm{n} . \mathrm{s})$ & $\mathrm{r}=0.102(\mathrm{n} . \mathrm{s})$ \\
\cline { 2 - 3 } Bruguiera cylindrica & $\mathrm{y}=-0.3488 \mathrm{x}+7749$ & $\mathrm{y}=0.1846 \mathrm{x}+4.2462$ \\
\hline \multirow{3}{*}{ Avicennia marina } & $\mathrm{r}=0.431(\mathrm{n} . \mathrm{s})$ & $\mathrm{r}=0.922 * *$ \\
\cline { 2 - 3 } & $\mathrm{y}=0.2319 \mathrm{x}+5.5241$ & $\mathrm{y}=0.8184 \mathrm{x}+1.989$ \\
\cline { 2 - 3 } & $\mathrm{r}=0.031(\mathrm{n} . \mathrm{s})$ & $\mathrm{r}=0.323(\mathrm{n} . \mathrm{s})$ \\
\cline { 2 - 3 } & $\mathrm{y}=-0.1733 \mathrm{x}+6.9771$ & $\mathrm{y}=1.9032 \mathrm{x}-6.3152$ \\
\hline
\end{tabular}

n.s = not significantly
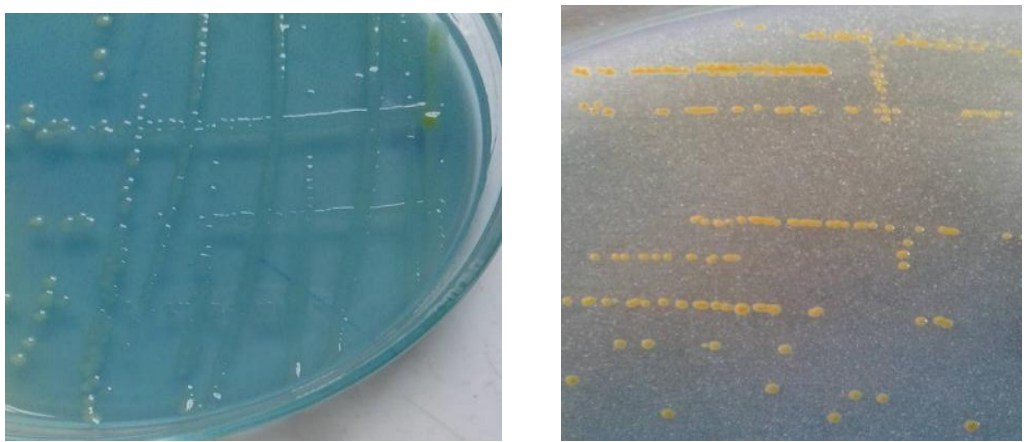

Figure 2a - The colonies of several isolates on Burk's $\mathrm{N}$ free media
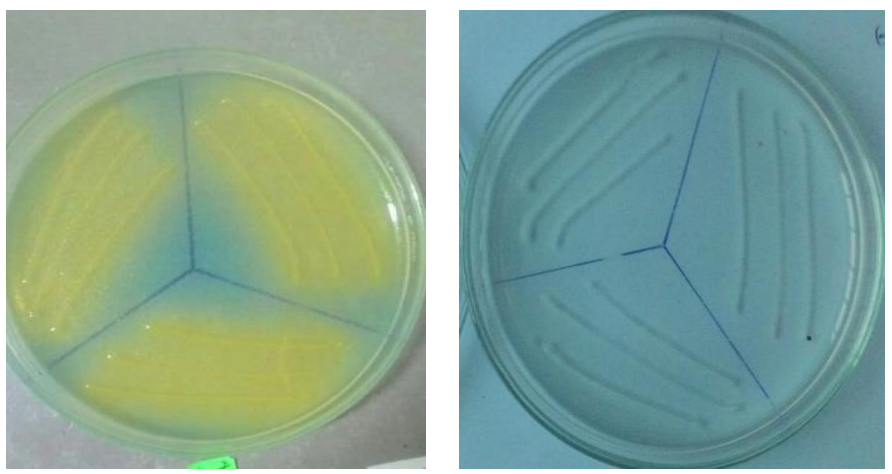

Figure $2 b$ - The colonies of four isolates in NBRIP medium with the halos around the colonies and changed the color of medium because of organic acids 
Almost their colonies have round-shaped; milky, white clear (on Burk's medium) and yellow, reddish yellow (on NBRIP medium); entire or loabate margin; diameter size of these colonies varied from 0.2 to $3.0 \mathrm{~mm}$ and all of them are Gram-positve and Gram-negative by Gram stain. Especially phosphate-solubilizing bacteria make a halo around colonies in NBRIP medium as according to Thanh and Diep [30], Tam and Diep [31] (Figure 2b).

The cells were observed by microscopic and appearded as short rods and most of them have motility.

Good isolates for nitrogen fixation (Rhizophora mucronata, Bruguiera cylindrica and Avicennia marina) were selected and presented in Table $3 \mathrm{a}, 3 \mathrm{~b}, 3 \mathrm{c}$.

Table 3a - Nitrogen fixation of good isolates $\left(\mathrm{mg} \mathrm{NH}_{4} / \mathrm{l}\right)$ from rhizosphere soil of Rhizophora mucronata $(15 / 37)$

\begin{tabular}{|c|c|c|c|c|c|}
\hline \multirow[t]{2}{*}{ No } & \multirow[t]{2}{*}{ Bacterial Isolates } & \multicolumn{4}{|c|}{ Rhizophora mисronata } \\
\hline & & Day 2 & Day 4 & Day 6 & Day 8 \\
\hline 01 & Control & $0.000 \mathrm{o}$ & $0.000 \mathrm{o}$ & $0.000 \mathrm{o}$ & $0.000 \mathrm{o}$ \\
\hline 02 & DDB1a & 0.3081 & 0.5421 & $0.000 \mathrm{o}$ & $2.763 \mathrm{~d}$ \\
\hline 03 & DDB1b & $0.024 \mathrm{n}$ & $0.053 \mathrm{n}$ & $2.728 \mathrm{~d}$ & $2.844 \mathrm{~cd}$ \\
\hline 04 & DDB2b & $0.046 \mathrm{n}$ & 0.2891 & $2.975 \mathrm{c}$ & $2.366 \mathrm{e}$ \\
\hline 05 & DDB2a1 & $0.045 \mathrm{n}$ & 0.3491 & $2.395 \mathrm{e}$ & 2.445 ef \\
\hline 06 & DDB2a2 & $0.054 \mathrm{n}$ & $0.739 \mathrm{jk}$ & $2.479 \mathrm{~d}$ & 2.388 ef \\
\hline 07 & DAB3a1 & 0.3381 & 0.4231 & $2.395 \mathrm{e}$ & $3.475 \mathrm{~b}$ \\
\hline 08 & DAB3b & $0.055 \mathrm{n}$ & $0.233 \mathrm{~m}$ & $3.409 \mathrm{~b}$ & $2.056 \mathrm{~g}$ \\
\hline 09 & DAB3a2 & $0.085 \mathrm{n}$ & 0.4221 & $2.976 \mathrm{c}$ & $2.511 \mathrm{~d}$ \\
\hline 10 & DLB4a & $0.017 \mathrm{n}$ & 0.4461 & $2.478 \mathrm{e}$ & $2.315 \mathrm{f}$ \\
\hline 11 & DLB4b & $0.085 \mathrm{n}$ & 0.3941 & $2.478 \mathrm{e}$ & $2.841 \mathrm{~cd}$ \\
\hline 12 & DAB5a & $0.095 \mathrm{n}$ & 0.3551 & $2.841 \mathrm{~cd}$ & $1.796 \mathrm{~h}$ \\
\hline 13 & DAB5b1 & $0.083 \mathrm{n}$ & $0.032 \mathrm{n}$ & $1.797 \mathrm{i}$ & $2.998 \mathrm{c}$ \\
\hline 14 & DAB5b2 & $0.029 \mathrm{n}$ & $0.039 \mathrm{n}$ & $1.998 \mathrm{~g}$ & $0.971 \mathrm{j}$ \\
\hline 15 & DLB6a & $0.085 \mathrm{n}$ & $0.241 \mathrm{~m}$ & $0.971 \mathrm{ij}$ & $0.782 \mathrm{jk}$ \\
\hline \multirow[t]{3}{*}{16} & DAB5c & 0.5841 & $4.156 \mathrm{a}$ & $0.786 \mathrm{jk}$ & $0.851 \mathrm{jk}$ \\
\hline & CV (\%) & \multicolumn{4}{|c|}{6.18} \\
\hline & F calculated & $* *$ & $* *$ & $* *$ & $* *$ \\
\hline
\end{tabular}

Table $3 \mathrm{~b}$ - Nitrogen fixation of good isolates $\left(\mathrm{mg} \mathrm{NH}_{4} / \mathrm{l}\right)$ from rhizosphere soil of Bruguiera cylindrica (17/45)

\begin{tabular}{|c|c|c|c|c|c|}
\hline \multirow[t]{2}{*}{ No } & \multirow[t]{2}{*}{ Bacterial Isolates } & \multicolumn{4}{|c|}{ Bruguiera cylindrica } \\
\hline & & Day 2 & Day 4 & Day 6 & Day 8 \\
\hline 01 & Control & $0.000 \mathrm{k}$ & $0.000 \mathrm{k}$ & $0.000 \mathrm{k}$ & $0.000 \mathrm{k}$ \\
\hline 02 & VDB1a & $0.075 \mathrm{hi}$ & $0.011 \mathrm{jk}$ & $0.236 \mathrm{c}$ & $0.027 \mathrm{j}$ \\
\hline 03 & VDB1b & $0.019 \mathrm{jk}$ & 0.0581 & $0.185 \mathrm{~d}$ & $0.021 \mathrm{jk}$ \\
\hline 04 & VDB2a & $0.018 \mathrm{jk}$ & 0.0651 & $0.187 \mathrm{~d}$ & $0.027 \mathrm{j}$ \\
\hline 05 & VDB2b & $0.028 \mathrm{j}$ & $0.073 \mathrm{hi}$ & $0.200 \mathrm{~d}$ & $0.011 \mathrm{jk}$ \\
\hline 06 & VDB2c & $0.085 \mathrm{~h}$ & $0.134 \mathrm{f}$ & $0.084 \mathrm{~h}$ & $0.032 \mathrm{j}$ \\
\hline 07 & VAB1a & $0.025 \mathrm{jk}$ & $0.018 \mathrm{jk}$ & $0.104 \mathrm{gh}$ & $0.025 \mathrm{j}$ \\
\hline 08 & VAB1b & $0.023 \mathrm{jk}$ & $0.459 \mathrm{a}$ & $0.032 \mathrm{j}$ & $0.019 \mathrm{jk}$ \\
\hline 09 & VAB1c & $0.034 \mathrm{j}$ & $0.034 \mathrm{j}$ & $0.154 \mathrm{e}$ & $0.022 \mathrm{jk}$ \\
\hline 10 & VAB1d & $0.032 \mathrm{j}$ & $0.011 \mathrm{jk}$ & $0.116 \mathrm{~g}$ & $0.016 \mathrm{jk}$ \\
\hline 11 & VAB2a & $0.056 \mathrm{i}$ & $0.115 \mathrm{~g}$ & $0.105 \mathrm{~g}$ & $0.017 \mathrm{jk}$ \\
\hline 12 & VAB2b & $0.054 \mathrm{i}$ & $0.343 \mathrm{~b}$ & $0.034 \mathrm{j}$ & $0.044 \mathrm{ij}$ \\
\hline 13 & VAB2c & $0.011 \mathrm{jk}$ & $0.016 \mathrm{jk}$ & $0.121 \mathrm{~g}$ & $0.020 \mathrm{jk}$ \\
\hline 14 & VLB1a & $0.032 \mathrm{j}$ & $0.101 \mathrm{~g}$ & $0.099 \mathrm{gh}$ & $0.014 \mathrm{jk}$ \\
\hline 15 & VLB1b & $0.322 \mathrm{~b}$ & $0.026 \mathrm{j}$ & $0.091 \mathrm{~h}$ & $0.027 \mathrm{j}$ \\
\hline 16 & VLB1c & $0.085 \mathrm{i}$ & 0.0661 & $0.112 \mathrm{~g}$ & $0.037 \mathrm{j}$ \\
\hline 17 & VLB2a & $0.033 \mathrm{j}$ & $0.019 \mathrm{jk}$ & $0.153 \mathrm{e}$ & $0.019 \mathrm{jk}$ \\
\hline \multirow[t]{3}{*}{18} & VLB2b & $0.192 \mathrm{~d}$ & $0.012 \mathrm{jk}$ & $0.296 \mathrm{~b}$ & $0.014 \mathrm{jk}$ \\
\hline & C.V (\%) & \multicolumn{4}{|c|}{16.68} \\
\hline & F calculated & $* *$ & $* *$ & $* *$ & $* *$ \\
\hline
\end{tabular}

Table 3c - Nitrogen fixation of good isolates $\left(\mathrm{mg} \mathrm{NH}_{4} / \mathrm{l}\right)$ from rhizosphere soil of Avicennia marina (15/43)

\begin{tabular}{|l|l|c|c|c|c|}
\hline No & Bacterial Isolates & \multicolumn{4}{|c|}{ Avicennia marina } \\
\cline { 3 - 5 } & & Day 2 & Day 4 & Day 6 & Day 8 \\
\hline
\end{tabular}




\begin{tabular}{|l|l|l|l|l|l|}
\hline 01 & Control & $0.000 \mathrm{~m}$ & $0.000 \mathrm{~m}$ & $0.000 \mathrm{~m}$ & $0.000 \mathrm{~m}$ \\
\hline 02 & MAN1a & $0.031 \mathrm{~m}$ & $1.993 \mathrm{~h}$ & $4.601 \mathrm{ab}$ & $0.042 \mathrm{~m}$ \\
\hline 03 & MAN1b & $0.032 \mathrm{~m}$ & $1.841 \mathrm{~h}$ & $4.322 \mathrm{c}$ & $0.041 \mathrm{~m}$ \\
\hline 04 & MAN2a & $0.061 \mathrm{~m}$ & $2.321 \mathrm{~g}$ & $4.393 \mathrm{c}$ & $0.031 \mathrm{~m}$ \\
\hline 05 & MAB2b & $0.032 \mathrm{~m}$ & $1.362 \mathrm{j}$ & $4.151 \mathrm{c}$ & $0.051 \mathrm{~m}$ \\
\hline 06 & MDB1a & $0.041 \mathrm{~m}$ & $1.181 \mathrm{j}$ & $4.433 \mathrm{bc}$ & $0.023 \mathrm{~m}$ \\
\hline 07 & MDN1b & $0.401 \mathrm{l}$ & $1.871 \mathrm{~h}$ & $4.751 \mathrm{a}$ & $0.053 \mathrm{~m}$ \\
\hline 08 & MDB1c & $0.033 \mathrm{~m}$ & $2.151 \mathrm{gh}$ & $4.552 \mathrm{~b}$ & $1.241 \mathrm{j}$ \\
\hline 09 & MDB2a & $0.071 \mathrm{~m}$ & $1.223 \mathrm{j}$ & $4.032 \mathrm{c}$ & $0.801 \mathrm{k}$ \\
\hline 10 & MDB2b & $0.082 \mathrm{~m}$ & $1.722 \mathrm{i}$ & $3.611 \mathrm{e}$ & $0.392 \mathrm{l}$ \\
\hline 11 & MLB1a & $0.041 \mathrm{~m}$ & $1.052 \mathrm{k}$ & $3.751 \mathrm{de}$ & $0.032 \mathrm{~m}$ \\
\hline 12 & MLB1b & $0.061 \mathrm{~m}$ & $1.482 \mathrm{j}$ & $4.311 \mathrm{c}$ & $0.042 \mathrm{~m}$ \\
\hline 13 & MLB2a & $0.062 \mathrm{~m}$ & $1.861 \mathrm{~h}$ & $4.151 \mathrm{c}$ & $1.611 \mathrm{~h}$ \\
\hline 14 & MLB2b & $0.151 \mathrm{~m}$ & $1.402 \mathrm{j}$ & $3.751 \mathrm{de}$ & $0.052 \mathrm{~m}$ \\
\hline 15 & MLB2c & $0.031 \mathrm{~m}$ & $2.461 \mathrm{~g}$ & $3.992 \mathrm{~d}$ & $0.053 \mathrm{~m}$ \\
\hline 16 & MLB2d & $2.831 \mathrm{f}$ & $2.021 \mathrm{~h}$ & $3.882 \mathrm{~d}$ & $0.191 \mathrm{~m}$ \\
\hline & \multicolumn{7}{|c|}{$\mathbf{6 . 5 6}$} \\
\hline & \multicolumn{1}{|c|}{ C calculated } & $* *$ & $* *$ & $* *$ & $*$ \\
\hline
\end{tabular}

Means within a column followed by the same letter/s are not significantly different at $\mathrm{p}<0.01$

Good isolates for phosphate solubilization (Rhizophora mucronata, Bruguiera cylindrica and Avicennia marina) were selected and presented in Table 4a, 4b, 4c

Table $4 \mathrm{a}$ - Phosphate solubilization $\left(\mathrm{mg} \mathrm{P}_{2} \mathrm{O}_{5} / \mathrm{l}\right)$ of good isolates from rhizosphere soil of Rhizophora mucronata (12/37)

\begin{tabular}{|c|c|c|c|c|c|}
\hline \multirow[t]{2}{*}{ No } & \multirow[t]{2}{*}{ Bacterial Isolates } & \multicolumn{4}{|c|}{ Rhizophora mucronata } \\
\hline & & Day 5 & Day 10 & Day 15 & Day 20 \\
\hline 01 & Control & $0.00 \mathrm{~s}$ & $0.00 \mathrm{~s}$ & $0.00 \mathrm{~s}$ & $0.00 \mathrm{~s}$ \\
\hline 02 & DDN1a1 & 101.99 op & 257.701 & $632.65 \mathrm{c}$ & $762.87 \mathrm{a}$ \\
\hline 03 & DDN1a2 & 141.47 no & $207.86 \mathrm{~m}$ & $370.53 \mathrm{i}$ & $377.84 \mathrm{i}$ \\
\hline 04 & DDN1b & $346.39 \mathrm{j}$ & $342.77 \mathrm{j}$ & $576.54 \mathrm{e}$ & $580.23 \mathrm{e}$ \\
\hline 05 & DDN1e2 & $21.04 \mathrm{rs}$ & $156.29 n$ & $463.29 \mathrm{~g}$ & $422.55 \mathrm{~h}$ \\
\hline 06 & DDN1e3 & $33.48 \mathrm{r}$ & $341.04 \mathrm{j}$ & $765.77 \mathrm{a}$ & $713.17 \mathrm{~b}$ \\
\hline 07 & DAN3a & $317.05 \mathrm{k}$ & $405.50 \mathrm{~h}$ & $621.05 \mathrm{~cd}$ & $689.42 \mathrm{~b}$ \\
\hline 08 & DAN3b1 & 272.811 & $390.41 \mathrm{i}$ & $555.21 \mathrm{ef}$ & $651.69 \mathrm{c}$ \\
\hline 09 & DLN4b & $162.98 \mathrm{~m}$ & 265.881 & $454.59 \mathrm{~g}$ & $717.56 \mathrm{~b}$ \\
\hline 10 & DDN1d1 & 139.32 no & $168.39 \mathrm{mn}$ & $388.13 \mathrm{i}$ & $535.32 \mathrm{f}$ \\
\hline 11 & DLN4c & 83.56 o & 260.691 & $427.88 \mathrm{~h}$ & $465.26 \mathrm{~g}$ \\
\hline 12 & DAN5d & $51.15 \mathrm{p}$ & $149.53 n$ & 288.131 & $415.76 \mathrm{hi}$ \\
\hline \multirow[t]{3}{*}{13} & DLN6a & $214.61 \mathrm{~m}$ & 266.261 & 259.421 & 269.091 \\
\hline & C.V (\%) & \multicolumn{4}{|c|}{4.87} \\
\hline & F calculated & $* *$ & $* *$ & $* *$ & $* *$ \\
\hline
\end{tabular}

Table $4 \mathrm{~b}$ - Phosphate solubilization $\left(\mathrm{mg} \mathrm{P}_{2} \mathrm{O}_{5} / \mathrm{l}\right)$ of good isolates from rhizosphere soil of Bruguiera cylindrica (13/45)

\begin{tabular}{|c|l|l|l|c|c|}
\hline \multirow{2}{*}{ No } & Bacterial Isolates & \multicolumn{4}{|c|}{ Bruguiera cylindrica } \\
\cline { 3 - 6 } & & \multicolumn{1}{|c|}{ Day 5} & Day 10 & Day 15 & Day 20 \\
\hline 01 & Control & $0.00 \mathrm{~s}$ & $0.00 \mathrm{~s}$ & $0.00 \mathrm{~s}$ & $0.00 \mathrm{~s}$ \\
\hline 02 & VDN1c & $387.86 \mathrm{l}$ & $340.78 \mathrm{~m}$ & $55.77 \mathrm{r}$ & $777.21 \mathrm{e}$ \\
\hline 03 & VDN1d & $337.17 \mathrm{~m}$ & $341.72 \mathrm{~m}$ & $43.66 \mathrm{r}$ & $925.05 \mathrm{c}$ \\
\hline 04 & VDN1f & $522.96 \mathrm{j}$ & $605.56 \mathrm{~h}$ & $350.84 \mathrm{l}$ & $1031.50 \mathrm{~b}$ \\
\hline 05 & VDN2a & $705.99 \mathrm{f}$ & $497.38 \mathrm{j}$ & $178.93 \mathrm{p}$ & $1003.35 \mathrm{~b}$ \\
\hline 06 & VDN2b & $639.17 \mathrm{~g}$ & $392.04 \mathrm{k}$ & $240.26 \mathrm{p}$ & $1020.12 \mathrm{~b}$ \\
\hline 07 & VDN2c & $507.52 \mathrm{j}$ & $373.32 \mathrm{l}$ & $94.96 \mathrm{q}$ & $848.06 \mathrm{~d}$ \\
\hline 08 & VDN2d & $382.64 \mathrm{l}$ & $370.49 \mathrm{l}$ & $106.65 \mathrm{q}$ & $975.41 \mathrm{c}$ \\
\hline 09 & VAN2a & $416.89 \mathrm{k}$ & $370.81 \mathrm{l}$ & $228.29 \mathrm{p}$ & $851.26 \mathrm{~d}$ \\
\hline 10 & VAN2d & $34.47 \mathrm{r}$ & $231.28 \mathrm{p}$ & $10.66 \mathrm{~s}$ & $777.21 \mathrm{e}$ \\
\hline 11 & VLN1b & $313.05 \mathrm{~m}$ & $283.39 \mathrm{n}$ & $3.72 \mathrm{~s}$ & $595.37 \mathrm{i}$ \\
\hline 12 & VLN1c & $655.76 \mathrm{~g}$ & $552.10 \mathrm{i}$ & $292.76 \mathrm{n}$ & $1091.18 \mathrm{a}$ \\
\hline
\end{tabular}




\begin{tabular}{|c|c|c|c|c|c|}
\hline 13 & VLN2c & $131.76 \mathrm{q}$ & $322.69 \mathrm{~m}$ & $382.24 \mathrm{k}$ & $445.16 \mathrm{k}$ \\
\hline 14 & VLN2e & $260.52 \mathrm{o}$ & $317.04 \mathrm{~m}$ & $229.29 \mathrm{rs}$ & $1073.41 \mathrm{a}$ \\
\hline & C.V (\%) & \multicolumn{4}{|c|}{3.40} \\
\hline & F calculated & $* *$ & $* *$ & $* *$ & $* *$ \\
\hline
\end{tabular}

Table $4 \mathrm{c}$ - Phosphate solubilization $\left(\mathrm{mg} \mathrm{P}_{2} \mathrm{O}_{5} / \mathrm{l}\right)$ of good isolates from rhizosphere soil of Avicennia marina (12/43)

\begin{tabular}{|c|c|c|c|c|c|}
\hline \multirow[t]{2}{*}{ No } & \multirow[t]{2}{*}{ Bacterial Isolates } & \multicolumn{4}{|c|}{ Avicennia marina } \\
\hline & & Day 5 & Day 10 & Day 15 & Day 20 \\
\hline 01 & Control & 0.001 & 0.001 & 0.001 & 0.001 \\
\hline 02 & MAB1b & $399.24 \mathrm{~d}$ & $134.06 \mathrm{ij}$ & $206.46 \mathrm{~h}$ & $154.63 \mathrm{i}$ \\
\hline 03 & MAB2b & $374.01 \mathrm{~d}$ & $70.12 \mathrm{k}$ & $132.28 \mathrm{j}$ & $178.84 \mathrm{i}$ \\
\hline 04 & MLB1b & $321.16 \mathrm{e}$ & $89.32 \mathrm{k}$ & $128.28 \mathrm{j}$ & $136.64 \mathrm{ij}$ \\
\hline 05 & MDN1a & $313.21 \mathrm{f}$ & $349.06 \mathrm{e}$ & $491.79 \mathrm{c}$ & $624.75 \mathrm{~b}$ \\
\hline 07 & MDN1b & $172.96 \mathrm{i}$ & $250.79 \mathrm{~g}$ & $475.43 \mathrm{c}$ & $703.79 \mathrm{a}$ \\
\hline 08 & MDN1d & $254.84 \mathrm{~g}$ & $295.76 \mathrm{e}$ & $488.20 \mathrm{c}$ & $600.40 \mathrm{~b}$ \\
\hline 09 & MDN2b & $206.14 \mathrm{i}$ & $357.24 \mathrm{e}$ & $492.99 \mathrm{c}$ & $745.71 \mathrm{a}$ \\
\hline 10 & MLN1b & $170.66 \mathrm{i}$ & $252.68 \mathrm{~g}$ & $497.86 \mathrm{c}$ & $715.57 \mathrm{a}$ \\
\hline 11 & MLN1c & $269.59 \mathrm{~g}$ & $264.78 \mathrm{~g}$ & $473.23 \mathrm{c}$ & $710.98 \mathrm{a}$ \\
\hline 12 & MLN1f & $251.15 \mathrm{~g}$ & $233.34 \mathrm{~g}$ & $428.92 \mathrm{~cd}$ & $473.65 \mathrm{c}$ \\
\hline \multirow[t]{3}{*}{13} & MLN2d & $183.72 \mathrm{i}$ & $253.31 \mathrm{~g}$ & $477.62 \mathrm{c}$ & $522.75 \mathrm{c}$ \\
\hline & C.V (\%) & \multicolumn{4}{|c|}{4.73} \\
\hline & F calculated & $* *$ & $* *$ & $* *$ & $* *$ \\
\hline
\end{tabular}

Means within a column followed by the same letter/s are not significantly different at $\mathrm{p}<0.01$

From these results (Table 3 and Table 4), almost abilities of bacteria in rhizosphere soil of mangrove were phosphate solubilization in comparison with nitrogen fixation. This may be explained that perhaps a possible nitrogen source has been contributed by decomposition of leaves of mangrove forest while the mangrove soils mainly contain inorganic phosphate so that they need to be solubilize this phosphos for growth.

Based on the good characteristics of these isolates (Table 3 and Table 4), 22 isolates were chosen to identify.

The fragments of 1495 bp 16S rRNA were obtained from PCR with 8F and 1492R primers and sequencing. Homology searches of 16S rRNA gene sequence of selected strain in GenBank by BLAST revealved that they had similarity to sequences of Bacilli (16/22 isolates) and 6 isolates belonged to Gamma-proteobacteria (Table 5).

Table 5 - Phylogenetic affiliation of isolates on the basis of 16S rRNA genes sequences by using BLAST programmes in the GenBank database based on sequences similarity.

\begin{tabular}{|c|c|c|}
\hline $\begin{array}{c}\text { Taxonomic Group and } \\
\text { Strain }\end{array}$ & Closest species relative & $\begin{array}{c}\text { Similarity } \\
(\%)\end{array}$ \\
\hline \multicolumn{3}{|c|}{ Bacilli } \\
\hline \multirow[t]{2}{*}{ MDB1c } & Bacillus sp. YY13 (KU298561) & 97 \\
\hline & Bacillus subtilis strain CR26 (KR780430) & 97 \\
\hline \multirow[t]{2}{*}{ MAB2b } & Bacillus subtilis strain BS-HOT1 (HM631977) & 98 \\
\hline & Bacillus sp. MN19(2014) (KM289136) & 98 \\
\hline \multirow[t]{2}{*}{ MAN1b } & Bacillus subtilis strain S12 (KU206485) & 98 \\
\hline & Bacillus sp. strain GY773 (KY473983) & 98 \\
\hline \multirow[t]{2}{*}{ MLN1b } & Bacillus subtilis strain OTEB48 (KP225283) & 99 \\
\hline & Bacillus sp. JN15 (KC121041) & 99 \\
\hline \multirow[t]{2}{*}{ MLN1c } & Bacillus sp. strain AU01 (MF590123) & 99 \\
\hline & Bacillus subtilis strain WJ-3 (JX673943) & 99 \\
\hline \multirow[t]{2}{*}{ MDN1a } & Bacillus flexus strain ML-27 (KJ401045) & 99 \\
\hline & Bacillus sp. P5'(2012) (JX083303) & 99 \\
\hline \multirow[t]{2}{*}{ MDN1b } & Bacillus subtilis strain MA-40 (KX426640) & 99 \\
\hline & Bacillus tequilensis strain V44.8fa (KT720325) & 99 \\
\hline \multirow[t]{2}{*}{ MDN2b } & Bacillus subtilis strain CR26 (KR780430) & 99 \\
\hline & Bacillus sp. strain YX48 (MF595820) & 99 \\
\hline \multirow[t]{2}{*}{ VLB2n } & Bacillus subtilis strain CR26 (KR780430) & 99 \\
\hline & Bacillus sp. strain YX48 (MF595820) & 99 \\
\hline
\end{tabular}




\begin{tabular}{|c|c|c|}
\hline VDB2a & Bacillus subtilis strain Md1-42 (MF581448) & 99 \\
\hline & Bacillus sp. strain WC5 (JN975953) & 99 \\
\hline \multirow[t]{2}{*}{ VDN2c } & Bacillus subtilis strain GX S-19 (KU904298) & 97 \\
\hline & Bacillus sp. strain 2N-14 (KX214613) & 97 \\
\hline \multirow[t]{2}{*}{ VDN1d } & Bacillus subtilis strain BJ-17 (GQ280027) & 99 \\
\hline & Bacillus sp. X15 (KP262341) & 99 \\
\hline \multirow[t]{2}{*}{$\mathrm{VAB} 2 \mathrm{~b}$} & Bacillus sp. strain Suaeda B-003 (KT981879) & 99 \\
\hline & Bacillus altitudinis strain WJB15 (KU877629) & 99 \\
\hline \multirow[t]{2}{*}{ DLB4b } & Bacillus subtilis strain CR26 (KR780430) & 99 \\
\hline & Bacillus sp. YY-14 (JX575605) & 99 \\
\hline \multirow[t]{2}{*}{ VDN1f } & Bacillus subtilis strain ZHA9 (FJ263018) & 99 \\
\hline & Bacillus sp. YY-14 (JX575605) & 99 \\
\hline \multirow[t]{2}{*}{ VAB1c } & Bacillus circulans strain MD1 (KT757520) & 99 \\
\hline & Bacillus sp. M-B (KC853425) & 99 \\
\hline \multicolumn{3}{|c|}{ Gammaproteobacteria } \\
\hline \multirow[t]{2}{*}{ MAB1b } & Enterobacter sp. WC141019 (KU245715) & 99 \\
\hline & Enterobacter cloacae strain BIA145 (KU161287) & 99 \\
\hline \multirow[t]{2}{*}{ VDN2d } & Enterobacter sp. strain Md1-52 (MF581458) & 99 \\
\hline & Enterobacter cloacae strain LC11-B (MF498495) & 99 \\
\hline \multirow[t]{2}{*}{ VAN2a } & Enterobacter sp. M3(2012) (JX081544) & 99 \\
\hline & Enterobacter cloacae strain LC11-B (MF498495) & 99 \\
\hline \multirow[t]{2}{*}{ MLN2d } & Vibrio sp. CR5 (KU052624) & 98 \\
\hline & Vibrio furnissii strain MM5 (FJ906812) & 98 \\
\hline \multirow[t]{2}{*}{ VLN2e } & Vibrio sp. QY27 (KP676706) & 98 \\
\hline & Vibrio fluvialis strain LCB1 (KC210808) & 98 \\
\hline \multirow[t]{2}{*}{ MLN1f } & Pseudomonas stutzeri strain W13 (KT380559) & 99 \\
\hline & Pseudomonas sp. IBUN MAR3 (DQ813309) & 99 \\
\hline
\end{tabular}

A neighbor-joining phylogenetic tree in these isolates showing the two clusters: cluster A divided into two cluster A1 and A2. Cluster A1 with cluster A11 had 5 isolates as Bacillus subtilis MAN1b, B. subtilis VDN1f, Bacillus sp. VAB2b, Bacillus flexus MDN1a and B. subtilis VLB2b1related very closely however they located into one cluster with strain Vibrio sp. VLN2e (one strain belongs to gram-negative bacteria) while cluster A12 with two strains Bacillus subtilis VDN1d and Bacillus circulans VAB1c had relationship close. Cluster A2 composed of two small clusters: cluster A21 with Bacillus sp. MUN1c and Vibrio sp. VLN2e, cluster A22 with Enterobacter sp. MAB1b and Enterobacter sp. VAN2a, both strains in cluster had relationship very close. Cluster B had cluster B1 with three strains: Bacillus subtilis MAB2b, B. subtilis VDB2a and Pseudomonas stutzeri MUN1f while cluster B2 composed of two small clusters: cluster B21 with Bacillus subtilis MDN1b, B. subtilis MUN1b and B. subtilis VDN2c related closely and cluster B22 with Bacillus sp. MDB1c and Enterobacter sp. located into one smaller cluter and Bacillus subtisis related B. subtilis DLB4b very closely. 


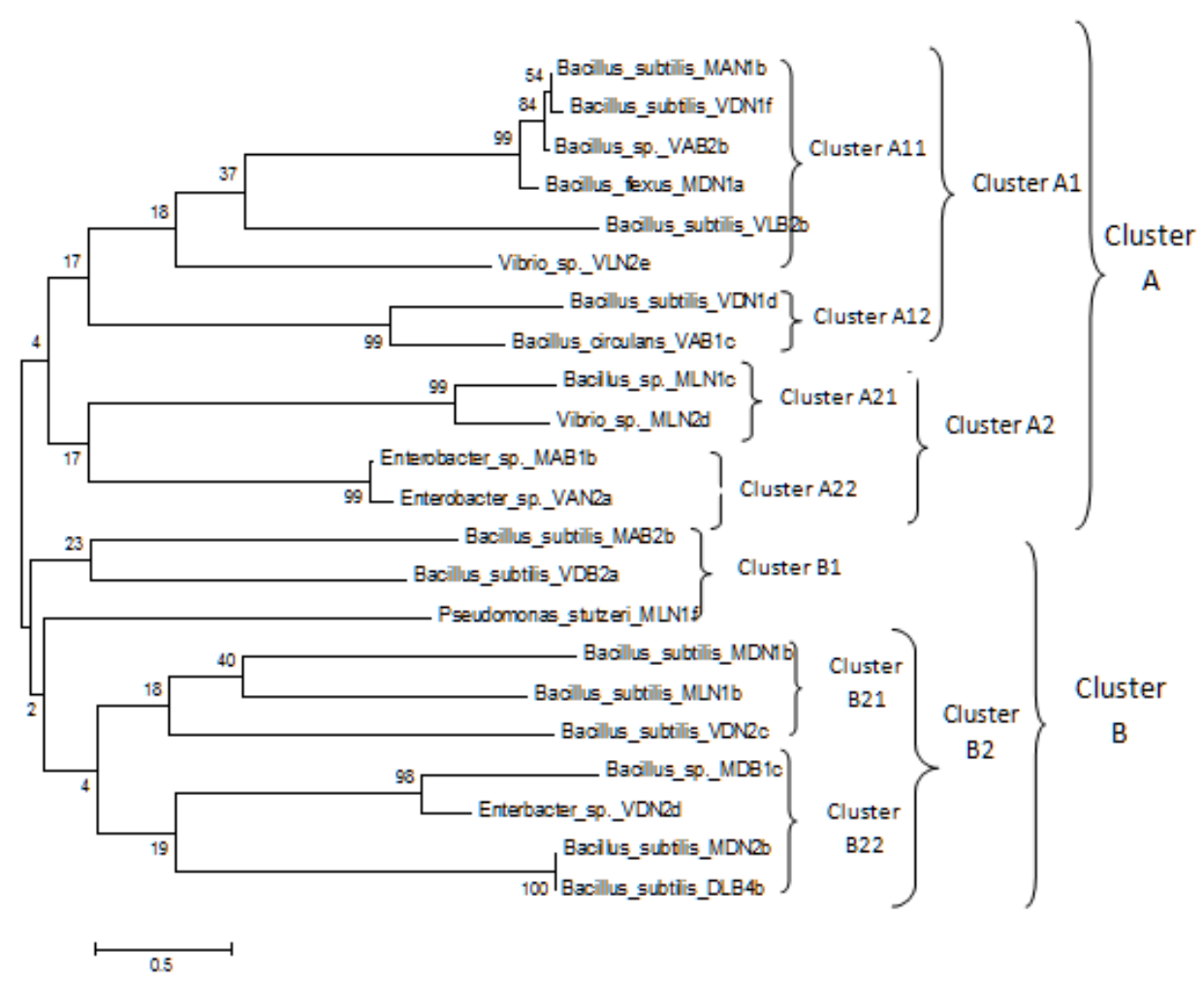

Figure 4 - Phylogenetic tree showing the relative position of rhizopheric bacteria (PGPR) by the neighborjoining method of complete 16S rRNA sequence. Bootstrap values of 1000 replicates are shown at the nodes of the trees.

The rhizospheric bacteria has been studied and described as beneficial bacteria with Gram-positive bacteria (Bacilli) presented on Burk's $\mathrm{N}$ free medium and it occupied over 70\% among 22 strains and 6 strains $(27 \%)$ belonged to Gammaproteobacteria in our result (Figure 5).

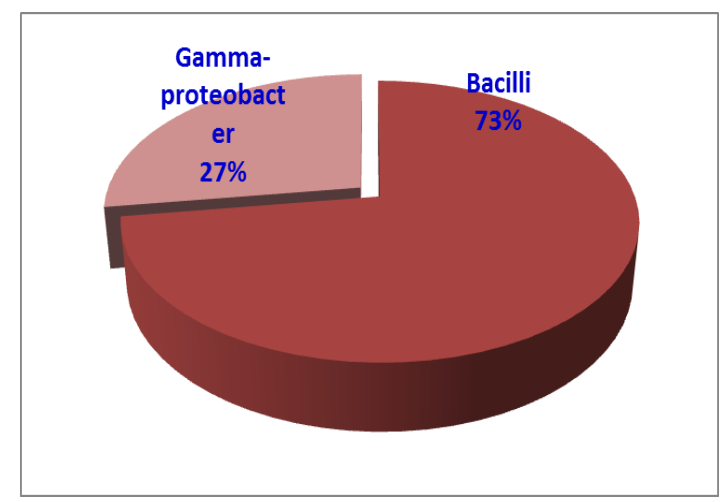

Figure 5 - The proportion of group and they distributed in two clusters

Almost rhizospheric bacteria from mangrove soil have ability of saline tolerant with $2.0 \% \mathrm{NaCl}$ however when increasing $\mathrm{NaCl}$ concentration from 2.5 to $4.0 \% \mathrm{NaCl}$ in the media, amount of bacteria reduced especially bacteria from rhizosphere of Rhizophora mucronata (Table 6) perhaps their roots developed from the air for a short time before they fell to the ground, and as a result, the strains from rhizosphere soil of Rhizophora mucronata were less salt tolerance than Bruguiera cylindrica and Avicennia marina which roots of two these kinds grew from saline soil. 
Table 6 - Ratio (\%) rhizospheric bacteria of mangrove soil with three kind of plant

\begin{tabular}{|c|c|c|c|c|c|}
\hline Ratio (\%) of Rhizospheric & \multicolumn{5}{|c|}{ NaCl concentration in medium (\%) } \\
\cline { 2 - 6 } Bacteria in three kinds of plant & $\mathbf{2 . 0}$ & $\mathbf{2 . 5}$ & $\mathbf{3 . 0}$ & $\mathbf{3 . 5}$ & $\mathbf{4 . 0}$ \\
\hline Rhizophora mucronata & 100 & 97.22 & 88.89 & 66.67 & 25.25 \\
\hline Bruguiera cylindrica & 100 & 100 & 100 & 86.67 & 86.17 \\
\hline Avicennia marina & 100 & 100 & 92.90 & 92.90 & 89.30 \\
\hline
\end{tabular}

Mangroves provide a unique ecological environment for diverse bacterial communities. Heterotrophic bacteria are very important in mangrove habitats as the bacteria decompose the mangrove litter, recycle the nutrients and produce the detritus food for many fishes [32][33]. Abundance and activities of the bacteria are controlled by various physicochemical parameters in the mangrove environment [34] [35] [36] [37] [38 [39 ]. Among heterotrophic bacteria, $\mathrm{N}_{2}$-fixing bacteria are efficient in using a variety of mangrove substrates [40].

Phosphorus $(\mathrm{P})$ is one of the essential elements for the growth and reproduction of bacteria and plays a very significant role in many aspects of cell metabolism. It is the second most important plant nutrient after nitrogen [41]. Phosphorous usually precipitate because of the abundance of cations in the interstitial water of mangrove sediments making phosphorus largely unavailable to plants, thus organisms that solubilise $\mathrm{P}$ can have important implications for plant growth, especially in nutrient-limited environments. This may be due to the fact that the bacteria are active in converting the insoluble forms of phosphorus compounds in mangrove soils to soluble forms that are readily transferred from soil to underlying water and or utilized by plants and microbes [42] [43] [44] [11] [12]. Muniyandi [45] has also observed higher level of phosphorus in the core mangrove areas than in the back mangrove areas. Venkateswaran [46] has reported that phosphate is efficiently absorbed by fine sediments of muddy areas than coarse areas. The changes in the levels of phosphorus can be linked with the influx of phosphorus from upstream regions and with regeneration into the overlying water column. Walsh [47] has reported that mud releases phosphates and nitrates during low saline conditions and it absorbs them from overlying water when it becomes more saline. In general, mangroves in low nutrient carbonate soils are limited by phosphorus, what phosphorus is present may be bound with calcium, efficiently holding it within the sediments [48].

Promod and Dhevendran [49] reported phosphate solubilisation by IPSB Vibrio sp. and Pseudomonas sp. of 0.5$0.55 \mathrm{mg} / \mathrm{l}$ from Cochin, India. Similarly seven bacterial sp such as, two Bacillus subtilis, three Pseudomonas sp. and two Azotobacter sp. reported from mangrove soil of Chollangi, East Godavari exhibited solubilising ability of $80-100 \mathrm{ug} / \mathrm{ml}$ of phosphate [50]. Genera of phosphate-solubilizing bacteria, like Pseudomonas, Bacillus, Corynebacterium, Vibrio, Micrococcus and Alcaligenes, were studied by Venkateswaran and Natarajan [51] in mangrove biotopes in Porto Novo, Chennai water and sediment. Our result found that Bacilli occupied over $70 \%$ of the total rhizospheric bacteria of mangrove soil, some good strain for nitrogen fixation as Bacillus subtilis DLB4b, Bacillus sp. VAB2b, Enterobacter sp. MAB1b and Bacillus sp. MDB1c and strains for phosphate solubilation as Bacillus subtilis MLN1b, Bacillus sp. MLN1c, Bacillus subtilis VDN1f and Bacillus subtilis MDN1c. All of them tolerated at a concentration of $4 \% \mathrm{NaCl}$ but strain Bacillus subtilis MAB2b is not only good nitrogen fixation but also high phosphate solubilization, so that it becomes a promising strain to produce biofertilizer for the crops which cultivated in soil salinity.

\section{CONCLUSION}

From 18 rhizosphere soil samples of three kinds of plants in mangrove forest as Rhizophora mucronata, Bruguiera cylindrica, and Avicennia marina, 125 isolates were isolated in two media (Burk's $\mathrm{N}$ free and NBRIP). They were identified as rhizospheric bacteria and 22 isolates having good plant growth promotion were chosen to analyse their relationship. These isolates were identified as Bacilli (more than 70\%) and Gammaproteobacteria on mangrove soil. Among them, one strain will be suggested to produce for crop cultivation on soil salinity in the future.

\section{ACKNOWLEDGEMENTS}

The authors thank the helpfulness of Microbiology BSc. Students and technicians in the Environment Microbiology Laboratory, Biotechnology R\&D Institute, Can Tho University, Vietnam. Ms. NGUYEN THI XUAN MY in the laboratory expriment and Ms. DAO THI MINH CHAU, Environment Microbiology Laboratory, Biotechnology R\&D Institute, Can Tho University, Vietnam for grammartical english.

\section{REFERENCES}

[1] S. Bhatt, D.G. Shah, and N. Desai, N, "The Mangrove Diversity of Purna Estuary, South Gujarat, India”. Tropical Ecology, vol. 50, pp. 287-293, 2009.

[2] K. Kathiresan, "How Do Mangrove Forests Induce Sedimentation?” Revista de Biologia Tropical, vol.51, pp.355-360, 2003.

[3] P.B. Tomlinson, “The Botany of Mangroves". Cambridge Tropical Biology Series. Cambridge University Press, Cambridge, 1986. 
[4] P. Saenger, "Mangrove Ecology, Silviculture and Conservation." Kluwer Academic Publishers, Dordrecht, pp.11-18, 2002. http://dx.doi.org/10.1007/978-94-015-9962-7.

[5] K. Kathiresan, "Global Policies and Institution. Biodiversity in Mangrove Ecosystems." In: Kathiresan, K. and Subramanian, A.N., Eds., UNU-UNESCO International Training Course on Biodiversity in Mangrove Ecosystems, Course Manual, Annamalai University, India, pp.317-332, 2003.

[6] V. Brinda, and A. Mathew, "Molecular Characterization and Identification of Unknown Bacteria from Waste Water." Indian Journal of Innovations and Developments, vol.1, pp.87-91, 2012.

[7] N.R. Pace, "A Molecular View of Microbial Diversity and the Biosphere." Science, vol. 272, pp.734-740, 1997. http://dx.doi.org/10.1126/science.276.5313.734.

[8] S. Malik, M, Beer, M, Megharaj, and R. Naidu, R, "The Use of Molecular Techniques to Characterize the Microbial Communities in Contaminated Soil and Water." Environment International, vol. 34, pp. 265-276, 2008. http://dx.doi.org/10.1016/i.envint.2007.09.001.

[9] P. Lakshmanaperumalsamy, D., Chandramohan, and R. Natarajan, R. "Studies on the nitrogen fixation by marine nitrogen fixing bacteria." Bulletin Department of Marine Science, University of Cochin. Vol.7, pp. 103-116, 1975.

[10] G. Holguin, M.A. Guzman, and Y. Bashan, "Two new nitrogen-fixing bacteria from the rhizosphere of mangrove trees: Their isolation, identification and in vitro interaction with rhizosphere Staphylococcus sp.” FEMS Microbiol. Ecol. Vol. 101. pp. 207-216, 1992.

[11] S. Ravikumar, "Nitrogen-fixing azotobacters from the mangrove habitat and their utility as biofertilizers." Ph.D. Thesis, Annamalai University, Parangipettai, India, 1975.

[12] P.Vazquez, G. Holguin, M.E. Puente, A. Lopez-Cortes, and Y. Bashan, Y. "Phosphate-solubilizing microorganisms associated with the rhizosphere of mangroves in a semiarid coastal lagoon." Biol. Feril. Soils. Vol. 30, pp. 460-468, 2000.

[13] Y. Bashan, and G. Holgiun, "Plant growth-promoting bacteria: a potential tool for arid mangrove reforestation." Trees. Vol.16, pp. $159-166,2002$

[14] F.D. Mann, and T.D. Steinke, "Biological nitrogen fixation (acetylene reduction) associated with decomposing Avicennia marina leaves in the Beach wood Mangroove " Nature Reserve. S. Afr. J. Bot. vol.58, pp.533-536, 1992.

[15] B.J. Hicks, and W.B. Silvester, "Nitrogen fixation associated with the New Zealand mangrove Avicennia marina (Forsk) Vierh. Var. resinifera (Forst. F) Bakh.” Appl. Environ. Microbiol. Vol.49, pp. 955-959, 1985.

[16] G. Toledo, Y. Bashan, and A. Soeldner, "Cyanobacteria and black Mangrooves in North Western Mexico. Colonization and diurnal and seasonal nitrogen fixation on aerial roots.” Can. J. Microbiol. Vol. 41, pp.,999-1011, 1995.

[17] M. Vassileva, R. Azcon, J. Barea Miguel, and N. Vassile, "Application of an encapsulated filamentous fungus in solubilisation of inorganic phosphate.” J. Biotechnol. vol. 63(1), pp.67-72, 1988.

[18] P. Gyaneshwar, G. Naresh Kumar, and L.J. Parekh, "Effect of buffering on the P-solubilizing ability of microorganisms." World J Microbiol. Biotechnol. vol.14, pp.669-673, 1998.

[19] V. Sundararaj, K. Dhevendran, D. Chandramohan, and K. Krishnamurthy, "Bacteria and primary production." Indian J. Mar. Sci. vol. 3, pp.139-141, 1974.

[20] M. Park, C. Kim, J. Yang, H. Lee, W. Shin, S. Kim and T. Sa, "Isolation and characterization of diazotrophic growth promoting bacteria from Gram rhizosphere of agricultural crops of Korea," Microbiological Research, vol 160, pp. 127-133, 2005.

[21] C.S. Nautiyal, "An efficient microbiological growth medium for screening phosphate-solubilizing microorganisms," FEMS Microbiology Letters, vol. 170, pp.256-270, 1999.

[22] H.J. Hoben, and P. Somasegaran, "Comparison of Pour, Spread and Drop Plate Methods for Enumeration of Rhizobium spp. In Inoculants made from presterilized peat," Appl. Environ. Microbiol., vol. 44, pp. 1246-1247, 1982.

[23] S. Turner, K.M. Pryer, V.P.M. Miao, and J.D. Palmer, "Investigating deep phylogenetic relationships among cyanobacteria and plastids by small subnit rRNA sequence analysis," J. Eukaryotic Microbiol., vol. 46, pp. 327-338, 1999.

[24] K. Tamura, D. Peterson, N. Peterson, G Stecher, M. Nei, and S. Kumar, "MEGA5: Molecular Evolutionary Genetics Analysis using Maximu m Likehood, Evolutionary Distance and Maximum Parsimony Methods," Mol. Biol. Evol., vol. 28, pp. 2731-2739, 2011.

[25] N. Rajendran, "Studies on mangrove-associated prawn seed resources of the Pichavaram mangrove, southeast coast of India." Ph.D. thesis, Annamalai University, Parangipettai, India, 1997.

[26] M. M. M. Selvam and K. Kathiresan, "Beneficial bacteria from soil of tropical mangrove" Asian Jr. of Microbiol. Biotech. Env. Sc. Vol. 12, No. (1), pp. 1-8, 2010.

[27] B. C. Behera, S. K. Singdevsachan, R. R. Mishra, B. K. Sethi, S. K. Dutta, H. N Thato, "Phosphate Solubilising Bacteria from Mangrove Soils of Mahanadi River Delta, Odisha, India" World Journal of Agricultural Research, Vol. 4, No. 1, pp.18-23, 2016. DOI:10.12691/wjar-4-1-3

[28] A.H. Goldstein, "Recent progress in understanding the molecular genetics and biochemestry of calcium phosphate solubilization by gram negative bacteria," Biological Agriculture and Horticulture, vol. 12., pp. 185-93., September 1995.

[29] K. Kpomblekou, and M.A.Tabatabai, "Effect of organic acids on release of phosphorus from phosphate rocks," Soil Science, vol. 158, pp. 442-453, December 1994.

[30] D.T..N. Thanh and C.N. Diep, "Isolation and Identification of rhizospheric bacteria in Acrisols of maize (Zea mays L.) in the eastern of South Vietnam. American J. Life Science. Vol. 2, No. 2, 2014, pp. 82-89. doi: 10.11648/j.ajls.20140202.18.

[31] H.M. Tam and C.N. Diep, "Isolation, Characterization and Identification of Endophytic Bacteria in Sugarcane (Saccharum spp. L.) Cultivated on Soils of the Dong Nai province, Southeast of Vietnam," American J. Life Science, vol.2, No.2 2014, pp. 361-368. doi: 10.11648/j.ails.2014206.16.

[32] W.E. and Odum E.J. Heald, Trophic analyses of an estuarine mangrove community. Bull Mar. Sci. vol. 22, pp.671-738,1972.

[33] A.D. Agate, C.V. Subramanian, and M. Vannucci, "Mangrove microbiology, Role of Microorganisms in nutrient cycling of mangrove soils and waters." Published by UNDP/UNESCO regional project of Asia and the Pacific (RAS/86/120) New Delhi, 1988.

[34] R. Palaniappan, and K. Krishnamurthy, "Heterotrophic bacteria of nearshore waters of the Bay of Bengal and Arabian Sea." Indian J. Mar. Sci., vol.14, pp.113-114, 1985. 
[35] L. Kannan, and K. Vasantha, "Distribution of heterotrophic bacteria in Vellar estuary, east coast of India.” Indian J. Mar. Sci. vol.15, pp. 267-268, 1986.

[36] K. Sathiayamurthy, R., BabuRajendran, A, Purushothaman, and V. Ramaiyan, "Heterotrophic bacteria from mangroves." Indian J. Microbiol. Vol.30, pp.337-341,1990.

[37] K. Kathiresan, and R. Veera, "Seasonal changes in tannin content of mangrove leaves." The Indian Foreste. Vol.116 (5), pp.390-392, 1990.

[38] R. Veera and K. Kathiresan, “Seasonal variation in gallotannin from mangroves.” Indian J. Mar. Sci. vol.19(3),pp. 224-225,1990.

[39] K. Kathiresan, P.,Moorthy, and S. Ravikumar, "Studies on root growth seedlings of a tropical mangrove tree species." International Tree Crops Journal. Vol.8 (2-3), pp.183-188, 1995.

[40] S.P. Pelegri, and R.R. Twilley, "Heterotrophic nitrogen fixation (acetylene reduction) during leaf-litter decomposition of two mangrove species from South Florida, USA. Mar. Biol. vol.131 (1), pp. 53-61, 1988.

[41] N.S. Subba Rao, "Advances in agricultural microbiology. In: Subba Rao NS, editor. Studies in the Agricultura and Food Sciences," Butterworth Scientific, London, 1982, pp.295-303.

[42] S. Niewolak, and A. Korycka, "Solubilization of basic clay by microorganisms in fertilized lakes." Pol. Arch. Hydrobiol. Vol.13, pp. 25-52, 1976.

[43] J.C.S. Lu, and K. Y. Chen, "Migration of chemical constituents in sediment-seawater interfaces." In: Chemistry of Marine Sediments. Edited by T.F. Yeu. Ann. Arbor Science Publ. U.S.A, 1977.

[44] M.J.H.A. Vander Linden, "Release of phosphorus from the sediment of polder ditches in a low moor peat area as estimated by core and enclosure experiments." Arch. Hydrobiol. Beih. Ergebn. Limnol. Vol.31, pp.373-381, 1988.

[45] K. Muniyandi, "Studies on mangrove of Pitchavaram (Southeast coast of India)." Ph.D. thesis, Annamalai University, India, 1985.

[46] K. Venkateswaran, "Studies on phosphate solubilizing and phosphatase producing bacteria from Porto Novocoastal waters." Ph.D. Thesis, Annamalai University, Parangipettai, India, 1981.

[47] G.C. Walsh, “An ecological Study of Hawaiian Mangrove Swamp. in Estuaries.” Edited by G.H. Lauff. Amer. Ass. Adv. Sci. (Pub.No.83). Washington, D.C. pp. 430-431, 1967.

[48] C.A.R. Silva, and A.A. Mozeto, "Release and retention of phosphorus in mangrove sediment, Sepetiba Bay, Brazil." In: Mangrove Ecosystem Studies in Latin America and Africa. Edited by B. Kjerfve, L.D. Lacerda, and S. Diop. UNESCO, Paris. pp. 179-190, 1997.

[49] K.C. Promod, and K. Dhevendaran, "Studies on phosphobacteria in Cochin backwater," Journal of Marine Biological Association of India,vol. 29. pp. 297-305, June 1987.

[50] A.V. Audipudi, N.P, Kumar, and A. Sudhir, "Phosphate solubilising mangrove associated with Chollangi mangrove soil in South east coast of India," International Journal of Scientific and Engineering Research, vol. 3(11). pp.1-9. November 2012.

[51] K. Venkateswaran K, and R. Natarajan, "Seasonal distribution of inorganic phosphate solubilising bacteria and phosphatase producing bacteria in Porto Novo waters.” Indian J Mar Sci. vol. 12(4), pp.:213-217, 1983. 